\title{
Prise en compte des effets gravitaires dans la modélisation du transport solide par charriage
}

\author{
Luc Hamm \\ SOGREAH Ingénierie \\ Jean-Michel Tanguy \\ Service Technique Central des Ports Maritimes et des Voies Navigables \\ Bainian Zhang \\ Université de Technologie de Compiègne
}

\begin{abstract}
Les formulations classiques de transport solide en régime permanent ont été établies sur des fonds plats ou à très faible pente pour lesquels les effets gravitaires dus à cette pente sont négligeables. Le développement des modèles numériques morphologiques pour les applications maritimes et fluviales a cependant mis en évidence l'importance de la prise en compte des effets gravitaires induits par la pente des fonds sur le transport solide. DE VRIEND et al. (1993) distinguent deux effets induits par cette prise en compte. Cela permet de stabiliser le calcul en empêchant les perturbations très locales du fond de se développer exponentiellement et d'aboutir, dans le cas d'un profil de plage, à un état d'équilibre dynamique stable.

Dans le domaine fluvial, l'étude de certaines morphologies telles que les rivières à fortes pentes, les formes de fonds (FrEDS $\emptyset$ E, 1974), les méandres (STRUiksma et al., 1985 ) ou les chenaux dragués (FREDS $\varnothing \mathrm{E}, 1978$ ) nécessitent également de considérer ce phénomène. Les recher-
\end{abstract}

ches publiées dans ce domaine ont couvert les études fondamentales de transport solide, les études des différentes morphologies citées et les aspects de modélisation numérique.

Dans le présent article, nous ferons d'abord un rapide tour d'horizon des études fondamentales de transport solide incluant l'effet gravitaire (chapitre 1). Puis une généralisation des formules de calcul de la contrainte critique de début d'entraînement est développée dans le chapitre 2. Le troisième chapitre traite de la comparaison de deux formulations du transport solide sur une pente longitudinale entre elles et avec des mesures en laboratoire effectuées par SMART (1984). Enfin le dernier chapitre présente les résultats de simulation numérique pour deux cas tests. Le premier concerne l'évolution d'une dune sur un fond plat. Le second traite de l'évolution des berges d'un chenal dragué dans l'axe du courant.

Taking into account gravitational effects when modelling sediment transport

This paper shows how existant formulae can be generalized for taking into account a slope with any angle and any direction when computing sediment transport. A first comparison with existing measures is presented. Numerical simulation issues show how important it is to take into account gravitational effects when computing movable beds evolutions. 


\section{Les études antérieures}

Les études des caractéristiques du transport solide sur une pente ont porté sur la modification de la contrainte critique de début d'entraînement et sur la modification de l'intensité et de la direction du transport par charriage. Elles ont toutes simplifié le problème en ne considérant que deux cas particuliers importants en pratique : celui d'une pente colinéaire à l'écoulement ou perpendiculaire à celui-ci.

\subsection{Cas d'une pente colinéaire à l'écoulement}

Dans le cas d'une pente colinéaire à l'écoulement, BAGNOLD (1956) a montré que la contrainte critique était modifiée de la façon suivante :

$$
\frac{\tau_{c r}}{\tau_{c r o}}=\frac{\sin \left(\theta_{t}+\phi\right)}{\sin \phi}=A_{t}
$$

$\tau_{c r}$ et $\tau_{c r o}$ sont les contraintes critiques sur la pente et sur fond plat respectivement, $\theta_{1}$ est l'angle que fait la pente avec l'horizontale et $\phi$ est l'angle de frottement interne du sédiment considéré.

Fernandez-Luque et VAN Beek (1976) ainsi que WhITEHOUSE (1991) ont vérifié expérimentalement la formule (1) en laboratoire. Ces auteurs ainsi que SMART (1984) ont également effectué des mesures de l'intensité du transport solide par charriage dans les conditions résumées dans le tableau $\mathrm{n}^{\circ} 1$ ci-dessous.

Tableau 1. - Mesures de transport solide sur une pente longitudinale en laboratoire

\begin{tabular}{|l|c|c|c|c|}
\hline \multicolumn{1}{|c|}{ MESURES DE TRANSPORT SOLIDE SUR UNE PENTE LONGITUDINALE EN LABORATOIRE } \\
\hline & $\begin{array}{c}\text { Pente } \\
\left(\tan \left(i_{1}\right)\right.\end{array}$ & $\begin{array}{c}d_{50} \\
(\mathrm{~mm})\end{array}$ & $\tau / \tau_{0}$ & $\begin{array}{c}\phi \\
\text { (degrés) }\end{array}$ \\
\hline $\begin{array}{l}\text { Fernandez-Luque and } \\
\text { Van Beek (1976) }\end{array}$ & 0 to 0.4 & 0.9 to 3.3 & 1.1 to 2.7 & 47 \\
\hline Smart (1984) & 0.03 to 0.2 & 2.0 to 10.5 & 2.7 to 33.0 & 33 \\
\hline Whitehouse (1991) & 0 to 0.62 & 1.2 & & 32 \\
\hline
\end{tabular}

\section{$d_{50}$ diamètre moyen du sédiment $\tau$ contrainte de cisaillement sur le fond $\phi$ angle statique de frottement solide.}

Ces mesures tendent à montrer que l'on peut utiliser une formule classique sur fond plat comme celle de MEYer-PETER et Mưller (1948) en remplaçant la contrainte critique d'entraînement sur fond plat par celle corrigée de l'effet de pente par l'équation (1). SMART (1984) note cependant que les formes de fonds sont beaucoup moins marquées sur un lit en pente que sur un lit plat, ce qui modifie le calcul de la contrainte effective qui seule participe au transport par charriage.

\subsection{Cas d'une pente transversale}

LANE (1955) a montré que la contrainte critique de début d'entraînement en présence d'une pente transversale s'écrivait :

$$
\frac{\tau_{c r}}{\tau_{\text {cro }}}=\cos \theta_{n}\left(1-\frac{\tan ^{2} \theta_{n}}{\tan ^{2} \phi}\right)^{1 / 2}=A_{n}
$$

où $\theta_{n}$ est l'angle de la pente transversale. Cette formule est obtenue dans le cas d'une portance négligeable. Elle a été vérifiée par des mesures en laboratoire effectuées par IKEDA (1982).

ENGELUND (1974) a proposé une méthode de calcul statique pour estimer la déviation de la direction du transport par rapport à celle de la contrainte de cisaillement.

L'équilibre des forces donne la relation:

$$
\sin \alpha=\frac{\operatorname{tg} \theta_{n}}{\operatorname{tg} \phi^{\prime}}
$$

$\alpha$ est l'angle de déviation et $\phi$ ' l'angle dynamique de frottement. Cette expression a été vérifiée expérimentalement par FREDS $\emptyset \mathrm{E}$ (1978). Une approche dynamique, plus réaliste physiquement, a été ensuite développée par ENGELUND (1981) en tenant compte de la vitesse relative des sédiments par rapport au courant. On obtient alors :

$$
\operatorname{tg} \alpha=\frac{0,6}{\sqrt{\tau !}}
$$

$\tau$ * est la contrainte adimensionnelle de cisaillement de peau.

Ce résultat a été vérifié expérimentalement avec les résultats de mesures en laboratoire de ZIMMERMANN et KENNEDY (1978).

\subsection{Cas général}

Le cas général d'un courant sur une pente d'inclinaison quelconque n'a quasiment pas été traité dans la littérature. 
VAN RUJN (1989) propose la correction suivante :

$$
\frac{\tau_{r r}}{\tau_{c r o r}}=A_{1} A_{n}
$$

Malheureusement, cette formule est incorrecte comme on peut le constater en considérant le cas limite d'un grain démarrant sous l'action gravitaire seule sur une pente dont I'inclinaison $\theta$ atteint l'angle $\phi$ de frottement interne (fig. I). On a alors :

$$
\operatorname{tg}^{2} \theta=\operatorname{tg}^{2} \theta_{x}+\operatorname{tg}^{2} \theta_{y}=\operatorname{tg}^{2} \varphi
$$

ce qui n'est pas en accord avec l'expression (5) dans laquelle le grain ne démarre seul que $\operatorname{si} \theta_{A}$ ou $\theta_{v}$ atteint la valeur $\phi$.

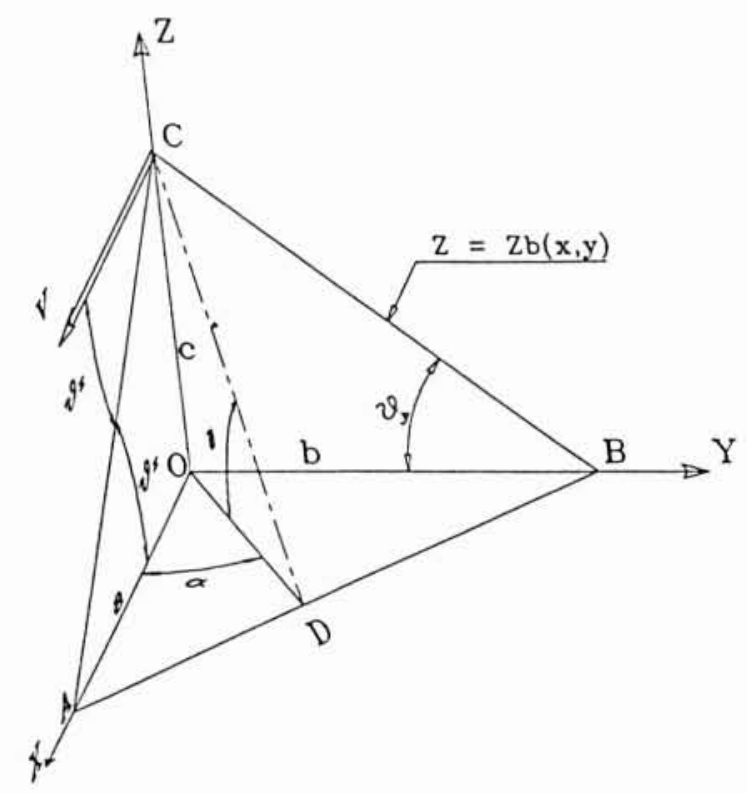

1. Equilibre d'un grain placé sur une pente.

Une approche plus numérique a également été proposée par Hauguel (1979), Struicksma et al. (1985) et WATANABE et al. (1986) pour estimer la modification de l'intensité et de la direction du transport solide. Elle consiste à modifier le calcul du transport solide sur fond plat en introduisant une composante de transport dans la direction de la pente soit :

$$
\vec{q}_{x}=\vec{q}_{s 0}-C\left(q_{s}\right) \overrightarrow{\operatorname{grad}} Z_{b}
$$

$q_{, 0}$ est le transport solide sur fond plat, $q_{\text {, }}$ est le transport solide sur la pente et $C\left(q_{s}\right)$ est une fonction qui peut être prise constante (HAUGuel, 1979) ou linéaire (STRUicksma et al. ; 1985. WATANABE et al. : 1986).

L'intérêt de cette approche est d'introduire un effet de diffusion dans l'équation de continuité des fonds qui stabilise le calcul. Du point de vue de la mécanique du transport solide, cette approche ne paraît pas vraiment justifiée comme nous le verrons au chapitre 3 .

\section{Le début d'entraînement sur une pente d'inclinaison quelconque}

Les formules rappelées précédemment peuvent se généraliser au cas d'une pente d'inclinaison quelconque en considérant l'équilibre d'un grain placé au point $C$ de la figure 1 . Ce grain est soumis aux forces d'entraînement et de portance induites par le courant que nous supposerons orientées selon l'axe $O X$ sans restreindre pour cela la généralité du raisonnement. Il est également soumis à la gravité et aux forces d'interaction avec les autres grains. Nous utiliserons ici la théorie mécanique du frottement sec pour représenter la liaison entre les grains participant au transport solide et ceux restant immobiles. Cette hypothèse de base signifie que nous ne regardons pas l'équilibre d'un seul grain mais d'une couche qui frotte sur une autre.

Pour exprimer mathématiquement l'équilibre vectoriel de ces forces, nous allons maintenant définir des repères de travail. On définit un système d'axes de référence $O X Y Z$, avec $O Z$ axe vertical orienté vers le haut et $O X Y$ plan horizontal. On associe à ce système d'axes un repère orthonormé absolu $(O, \vec{i}, \vec{j}, \vec{k})$. Dans ce système d'axes, le plan incliné a pour équation $Z=Z_{b}(X, Y)$. On définit alors les angles $\theta_{x}$ et $\theta_{v}$ par :

$$
\operatorname{tg} \theta_{1}=\frac{\partial Z_{b}}{\partial x} ; \quad \operatorname{tg} \theta_{y}=\frac{\partial Z_{b}}{\partial y} .
$$

Considérons maintenant un repère lié au mouvement du grain sur le plan incliné $(O, \vec{l}, \vec{J}, \vec{K})$ avec $\vec{K}$ vecteur normal au plan incliné et $\vec{l}$ contenu dans le plan OXZ.

On peut passer du repère absolu à ce repère lié au plan par deux rotations: la première d'axe $(O, \vec{J})$ et d'angle $\theta_{1}=-\theta_{x}$ et la seconde d'axe $(O, \vec{l})$ et d'angle $\theta_{2}$ définie par :

$$
\operatorname{tg} \theta_{2}=-\operatorname{tg} \theta_{y} \cos \theta_{y} .
$$

Nous écrivons maintenant dans le repère lié l'équilibre statique des cinq forces qui s'exercent sur le sédiment, soit :
$\vec{F}_{c}=A_{c} \tau_{b} \vec{l}$
force d'entraînement
$\vec{F}_{p}=A_{p} \tau_{b}, \vec{K}$
force de portance
$\vec{G}=-G \vec{k}$
gravité apparente
$\vec{R}=R \vec{K}$
réaction du sol sur les grains
$\vec{F}_{1}=-R \operatorname{tg} \phi \times$
$\times(\cos \alpha \vec{l}+\sin \alpha \vec{J})$
force de frottement qui s'oppose au mouvement

avec $\alpha$ l'angle de déviation du transport par rapport au courant lors du début d'entraînement.

La force de portance est considérée ici comme orientée perpendiculairement au plan du transport conformément aux résultats de FERNANDEZ-LUQUE et VAN BEEK (1976), L'équilibre des forces au début du mouvement, pour lequel $\tau_{b}=\tau_{i}$, s'écrit :

$$
\begin{aligned}
\left(A_{c} \tau_{c r}-R \operatorname{tg} \varphi \cos \alpha\right) \vec{I} & +\left(R+A_{p} \tau_{c r}\right) \vec{K}- \\
& -G \vec{k}-R \operatorname{tg} \phi \sin \alpha \vec{J}=0
\end{aligned}
$$


avec

$$
\vec{k}=\sin \theta_{1} \vec{I}+\cos \theta_{1} \sin \theta_{2} \vec{J}+\cos \theta_{1} \cos \theta_{2} \vec{K} .
$$

En projetant sur l'axe $(O, \vec{K})$, on obtient la réaction $R$, soit :

$$
R=G \cos \theta_{1} \cos \theta_{2}-A_{p} \tau_{c r} .
$$

En projetant ensuite sur l'axe $(O, \vec{J})$, on obtient l'angle de déviation $\alpha$, soit :

$$
G \vec{k} \cdot \vec{J}+R \operatorname{tg} \varphi \sin \alpha=0
$$

soit :

$$
\sin \alpha=-\frac{G \sin \theta_{2} \cos \theta_{1}}{\left(G \cos \theta_{1} \cos \theta_{2}-A_{p} \tau_{c r}\right) \operatorname{tg} \varphi}
$$

ou encore :

$$
\sin \alpha=-\frac{\operatorname{tg} \theta_{2}}{\operatorname{tg} \varphi}\left(1-\frac{A_{p} \tau_{c r}}{G \cos \theta_{1} \cos \theta_{2}}\right)^{-1} .
$$

Enfin, en projetant sur l'axe $(O, \vec{I})$, on obtient :

$$
A_{e} \tau_{c r}-R \operatorname{tg} \phi \cos \alpha-G \sin \theta_{1}=0
$$

soit :

$$
\begin{aligned}
A_{e} \tau_{c r}=G & \sin \theta_{1}+ \\
& +\left(G \cos \theta_{1} \cos \theta_{2}-A_{p} \tau_{c r}\right) \operatorname{tg} \phi \cos \alpha .
\end{aligned}
$$

En prenant $\theta_{1}=\theta_{2}=0$, on obtient également :

$$
A_{e} \tau_{c r o}=\left(G-A_{p} \tau_{c r o}\right) \operatorname{tg} \phi
$$

soit :

$$
\tau_{c r o}=\frac{G \operatorname{tg} \varphi}{A_{e}+A_{p} \operatorname{tg} \varphi} .
$$

L'expression (13) peut se simplifier en supposant que la déviation du transport reste suffisamment faible pour que l'expression $\left(A_{e}+A_{p} \operatorname{tg} \phi \cos \alpha\right)$ soit très proche de $\left(A_{e}+A_{p} \operatorname{tg} \phi\right)$. On obtient alors :

$$
\tau_{c r}=\tau_{c r o}\left(\cos \theta_{1} \cos \theta_{2} \sqrt{1-\frac{\operatorname{tg}^{2} \theta_{2}}{\operatorname{tg}^{2} \Phi}}+\frac{\sin \theta_{1}}{\operatorname{tg} \varphi}\right) \text {. }
$$

Cette expression se ramène effectivement à l'expression (1) en cas de pente colinéaire à l'écoulement $\left(\theta_{2}=0\right)$, et à l'expression (2) dans le cas de pente perpendiculaire à l'écoulement $\left(\theta_{1}=0\right)$. Soulby (1993) a également récemment démontré ce résultat en choisissant un repère lié à la ligne de plus grande pente. En écrivant que la contrainte critique doit rester positive ou nulle, on retrouve également l'expression (6). Enfin, la déviation du transport solide peut se calculer par l'équation (12). Cela correspond à l'approche statique de ENGELUND (1974).

\section{Influence d'une pente longitudinale sur le transport solide par charriage}

Dans le présent chapitre, deux formulations de l'influence de la pente des fonds sur le transport solide par charriage décrites dans la littérature sont comparées. Leur équivalence est démontrée sous certaines conditions. Puis une validation est effectuée par comparaison avec des mesures en laboratoires effectuées par SMART (1984).

\subsection{Comparaison des formulations utilisées}

La formule de MeYer-Peter et MÜLler (1948) a été utilisée pour ces calculs sous trois formes:

- La formule classique à fond plat sans formes de fond (version fond plat) qui s'écrit:

$$
q_{s} \cdot 0=8(\tau \cdot-\tau \cdot c r o)^{3 / 2} .
$$

- Modification de la contrainte critique de cisaillement pour tenir compte de l'effet de pente (version «pente $1 »)$ :

$$
q_{s * 1}=8\left(\tau .-A_{t} \tau_{* r o}\right)^{3 / 2} .
$$

- Modification d'un transport solide par l'ajout de l'influence de la pente (version "pente $2 »)$ sous la forme :

$$
q_{s * 2}=q_{s * 0}\left(1-C \operatorname{tg} \theta_{t}\right)
$$

$q_{s^{*}}$ est le transport solide adimensionnel, $\tau$. est la contrainte de cisaillement adimensionnelle, $\tau_{*_{\text {cro }}}$ et $\tau \tau_{c r}$ sont les contraintes critiques adimensionnelles sur fond plat et en présence d'une pente, $\theta_{1}$ est l'angle que fait le fond avec l'horizontale et $\phi$ est l'angle de frottement interne du sédiment, $C$ est une constante à déterminer.

Une première comparaison purement numérique a été effectuée en choisissant une valeur de $C$ égale à 0,5 et une valeur de $\phi$ égale à $33^{\circ}$. Le ratio transport solide sur pente sur transport solide sur fond plat a été calculé en fonction de l'angle de la pente pour une large gamme de contraintes de cisaillement. Les résultats sont illustrés sur la figure 2.

On observe dans ce cas que ce ratio est quasiment linéaire pour la formule (pente 2) ce qui s'explique par le fait que pour les pentes considérées, la tangente de l'angle n'est pas éloignée de la valeur de celui-ci.

Les deux formulations donnent des résultats très différents sauf lorsque $\tau$. vaut environ 0,2 à 0,3 . La forme des

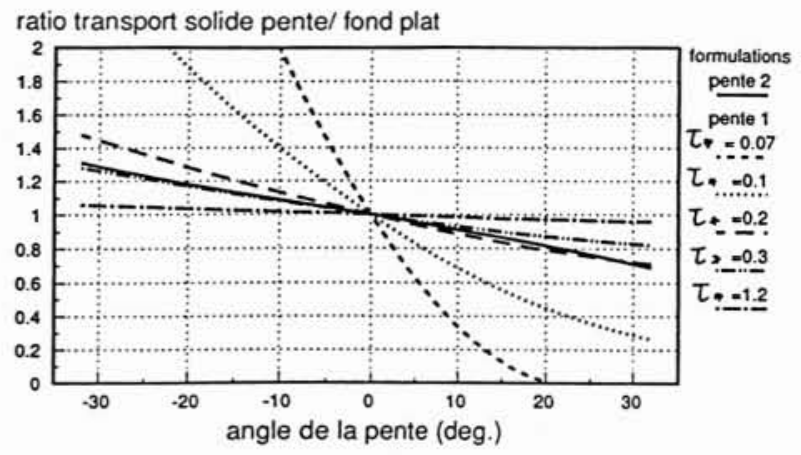

2. Comparaison des formules du transport solide en présence d'une pente longitudinale. 
courbes laisse à penser que la formule (18) serait tangente à la formule (17) si le coefficient $C$ était variable. Cette observation peut se démontrer de la façon suivante: l'équation (17) peut s'écrire :

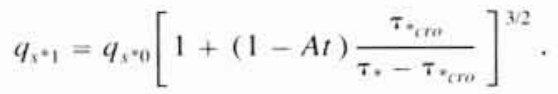

En supposant que le second terme entre crochets est suffisamment petit devant 1 , on peut écrire au premier ordre :

$$
q_{s * 1}=q_{s+0}\left[1-\frac{3}{2}(A,-1) \frac{\tau_{c_{c r o}}}{\tau \cdot-\tau_{* r p}}\right] .
$$

Or D'où :

$A_{t}-1=\operatorname{tg} \theta / \operatorname{tg} \phi$ à l'ordre $2($ avec $\cos \theta=1)$.

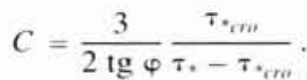

Si $C=0,5$, on trouve alors $\tau$. $=0,265$ ce qui confirme l'analyse des figures précédentes. L'équivalence des deux formulations est donc démontrée dans le cas où la pente est faible et où on est assez loin du seuil de démarrage. Dans le cas où $C$ est une constante, on peut obtenir des écarts importants lorsque l'on varie sur une large gamme de $\tau$. comme l'illustre la figure 2 . Il faut noter également que la relation (22) sera modifiée si une formule différente de transport solide est utilisée.

\subsection{Comparaison avec les mesures de SMART (1984)}

SMART (1984) rapporte quatre séries d'expériences effectuées en laboratoire sur un canal inclinable dans les conditions résumées dans le tableau $\mathrm{n}^{\circ} 2$ ci-dessous. II faut noter que les pentes de fonds testées sont restées assez loin de la pente d'équilibre du sédiment $\left(\theta_{t \max }=11,3^{\circ}\right.$ à comparer à $\left.\phi=33^{\circ}\right)$ et que les contraintes de cisaillement étaient assez éloignées du seuil de démarrage. On se trouve donc ici précisément dans les conditions où les deux formulations sont équivalentes.

Tableau 2. - Essais de Smart (1984). Conditions expérimentales.

\begin{tabular}{|l|c|c|c|c|c|c|c|c|c|}
\hline \multicolumn{10}{|c|}{ Essais de Smart (1984) - Conditions expérimentales } \\
\hline \multicolumn{1}{|c|}{ Titre du test } & $\begin{array}{c}d_{m} \\
(\mathrm{~mm})\end{array}$ & $\begin{array}{c}d_{90} \\
(\mathrm{~mm})\end{array}$ & $d_{90} / d_{30}$ & $\begin{array}{c}Q_{s} \\
\left(\mathrm{~kg} / \mathrm{m}^{3}\right)\end{array}$ & $\tau *_{c r o}$ & $\begin{array}{c}\phi \\
\left({ }^{\prime}\right)\end{array}$ & $\operatorname{tg} \theta$ & $\tau^{\prime} \tau_{c r}$ & $\tau \cdot$ \\
\hline $\begin{array}{l}\text { Granulométrie uniforme } \\
4,2 \mathrm{~mm}\end{array}$ & 4,2 & 5,2 & 1,44 & 2670 & 0,05 & 33 & 0,05 à 0,2 & 6 à 0,54 & 0,3 à 1,8 \\
\hline $\begin{array}{l}\text { Granulométrie uniforme } \\
10,5 \mathrm{~mm}\end{array}$ & 10,5 & 12,1 & 1,34 & 2680 & 0,05 & 33 & 0,034 à 0,2 & 3 à 22 & 0,1 à 0,9 \\
\hline $\begin{array}{l}\text { Granulométrie étalée } \\
2,0 \mathrm{~mm}\end{array}$ & 2,0 & 4,6 & 4,60 & 2680 & 0,039 & 33 & 0,05 à 0,2 & 21 à 124 & 0,8 à 3,3 \\
\hline $\begin{array}{l}\text { Granulométrie étalée } \\
4,3 \mathrm{~mm}\end{array}$ & 4,3 & 11,0 & 8,46 & 2670 & 0,05 & 33 & 0,03 à 0,2 & 4 à 69 & 0,2 à 2,3 \\
\hline
\end{tabular}

La méthodologie des essais est décrite en détail dans SMART (1984) auquel nous reportons le lecteur. Les résultats fournis incluent le débit liquide corrigé, le débit solide, la hauteur d'eau corrigée, la contrainte de cisaillement, le coefficient de STRICKLER corrigé et le coefficient de transport effectif. Les grandeurs corrigées citées ont fait l'objet d'une correction pour éliminer l'effet des parois latérales. La largeur du canal était de $0,2 \mathrm{~m}$. La contrainte critique d'entraînement en présence d'une pente n'a pas été mesurée mais calculée par la formule (3) avec un angle de frottement interne estimé à $33^{\circ}$.

Chaque condition d'essai a fait l'objet de calculs à l'aide des trois formules. Une valeur de $C$ égale à 0.5 a été choisie pour la formule (18). La comparaison a été effectuée en traçant les droites de régression linaire reliant transport mesuré et calculé (voir fig. 3). On note que les variantes de la formule de MEYer-PETER et MÜLLER sont très proches. La comparaison avec les mesures est plutôt bonne en granulométrie uniforme. En granulométrie étendue, les formules sous-estiment systé- matiquement le transport. Cela indique la présence d'un transport en suspension important. SMART reconnaît luimême la présence de transport en suspension dans ses essais. Cette difficulté relativise d'ailleurs l'intérêt de la formule empirique de transport solide proposée par SMART (1984) en granulométrie étendue. Cette formule a en effet été établie à partir d'une régression sur l'ensemble des mesures et inclut donc une part indéterminée de transport en suspension. L'accord le meilleur est obtenu pour la série de tests en granulométrie uniforme $10,5 \mathrm{~mm}$ pour laquelle la suspension doit être très faible.

Ainsi, la comparaison avec des mesures en laboratoire a montré que l'inclusion de l'effet de pente dans la formule de MEYER-PETER et MÜLLER n'améliorait pas vraiment la corrélation. Du point de vue morphologique, cette influence est cependant prépondérante comme nous allons maintenant le montrer. Il conviendrait également d'effectuer à l'avenir une comparaison dans le cas où la contrainte d'entraînement est proche du seuil de démarrage ce qui n'est pas le cas des essais de SMART. 
Granulométrie uniforme $4.2 \mathrm{~mm}$

transport calculé

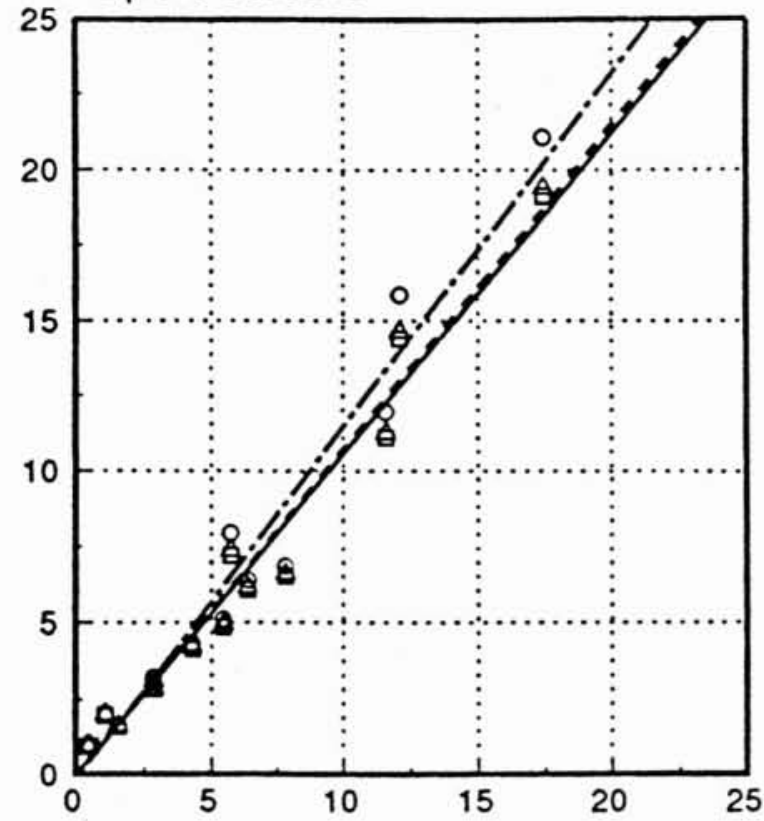

transport adimensionnel mesuré

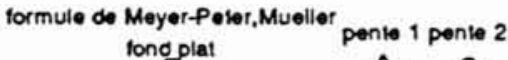

tonoplat
Granulométrie uniforme $10.5 \mathrm{~mm}$ transport calculé

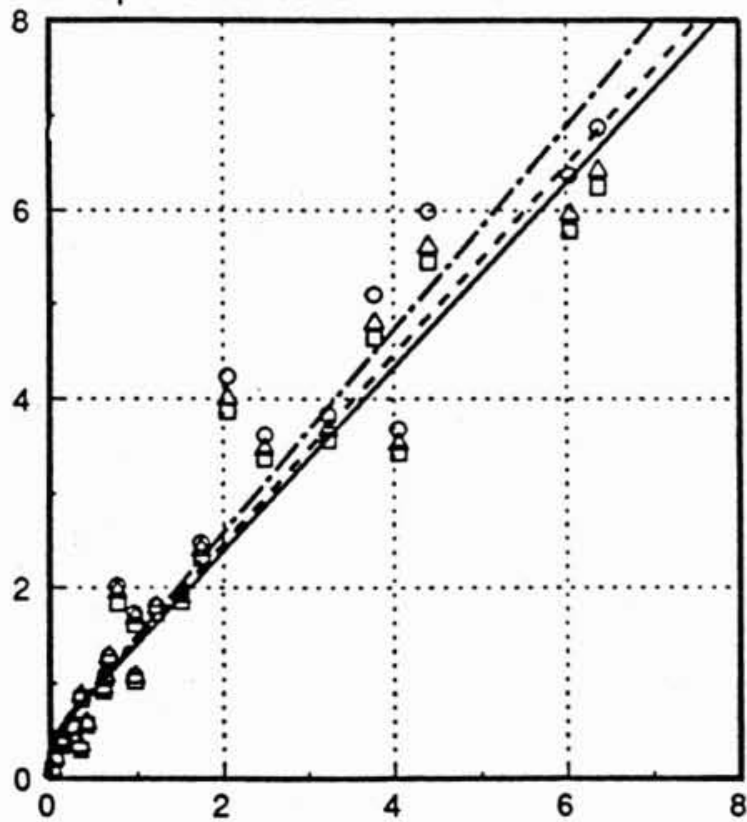

transport adimensionnel mesuré

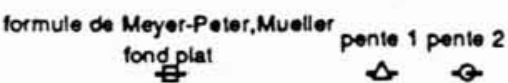

Granulométrie étalée $2.0 \mathrm{~mm}$ transport calculé

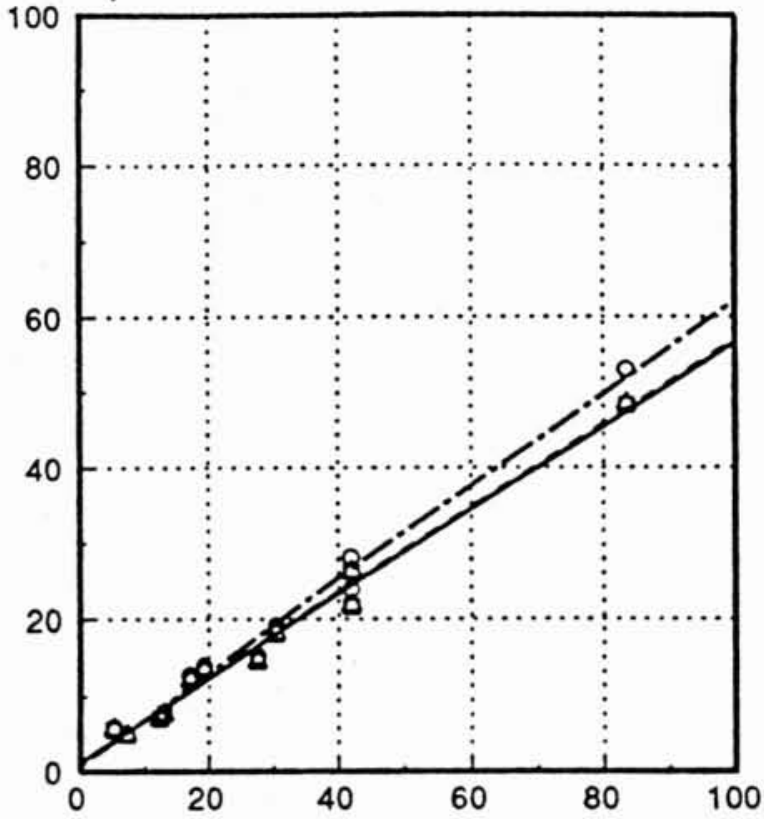
transport adimensionnel mesuré

formule de Meyer-Peter, Mueller tond plat
Granulométrie étalée $4.3 \mathrm{~mm}$ transport calculé

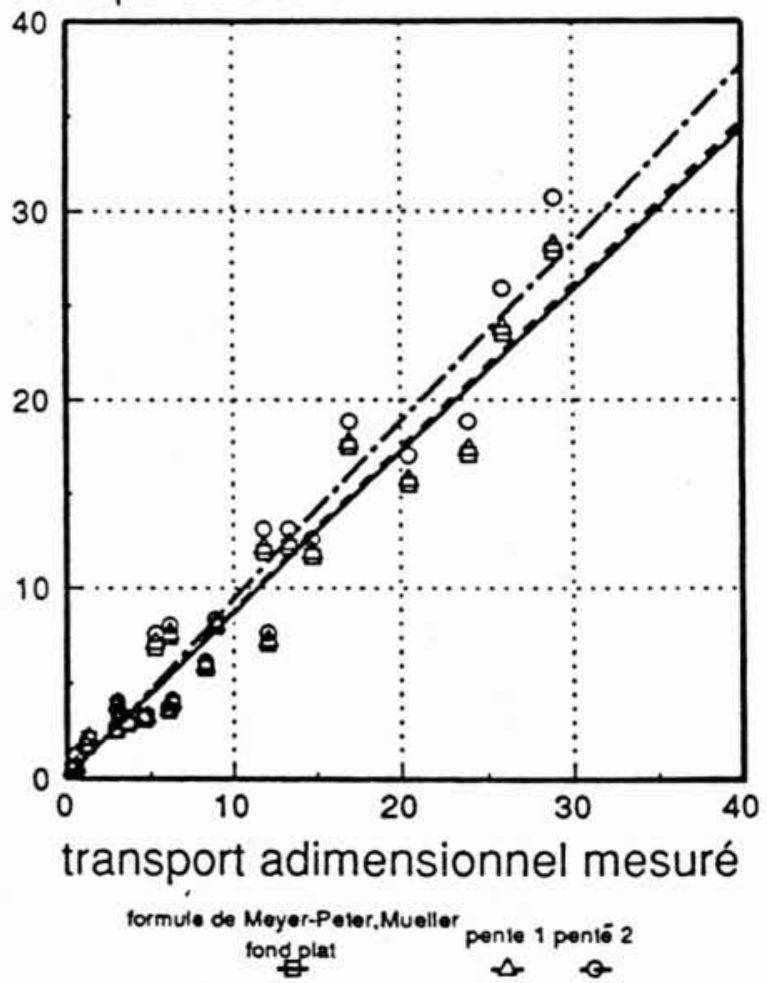

3. Essais de SMART (1984). Comparaison des valeurs mesurées et calculées pour les quatre séries de tests. 